Article

\title{
PREPARATION AND CHARACTERIZATION OF A BIOARTIFICIAL POLYMERIC SYSTEM (SCAFFOLD) MADE OF CHITOSAN AND POLYVINYL ALCOHOL WITH POTENTIAL IN THE VIABILITY OF BONES CELLS
}

Sebastián Ramirez Tarazona ${ }^{1}$, Juan Ochoa Melo ${ }^{1}$, Julián Ángel García ${ }^{1}$, Andres Bernal Ballén ${ }^{1}$, Hugo Ramiro Segura Puello ${ }^{2}$, Andrea Villamil Ballesteros ${ }^{2}$, Diana Nieto Mosquera ${ }^{2}$ and Diana Muñoz Forero ${ }^{2}$

1 Biomedical Engineering Research Group, Manuela Beltran University, Avenida Circunvalar No 60-00, Bogotá 110231, sebastianramirez.t@academia.umb.edu.co

2 Laboratory of Health Research, Manuela Beltran University, Avenida Circunvalar No 60-00, Bogotá 110231

Abstract: Scaffolds are widely used in tissue engineering because their manufacture is based on natural and synthetic polymers, which allows them to have properties such as biocompatibility and biodegradability, creating an ideal environment for cell growth on their surface. In this context, among the polymers studied in Tissue Engineering are Chitosan ( $\mathrm{CH}$ ) and Polyvinyl Alcohol (PVA). $\mathrm{CH}$ is a versatile polymer obtained from deacetylation of chitin, which is used for its high biodegradability and biocompatibility, although its mechanical properties must be improved. It has been found that one of the ways to improve the mechanical properties of $\mathrm{CH}$ is to mix it with other synthetic polymers such as PVA. PVA is known for its biocompatibility, biodegradability, zero toxicity and ease of preparation due to its solubility in water and excellent mechanical properties, such as tensile strength and ease in the formation of films and barriers. In this study we evaluated the capacity of scaffolds made with $\mathrm{CH}$ and PVA in different concentrations (2: 1, 1: 1, 1: 2, respectively) as a possible application in bone regeneration. This was made through different characterization tests such as Infrared Spectroscopy, AFM, Swelling test and Porosity test, where we obtained information about its structural and physicochemical properties. Additionally, a cellular quality control was performed on the material through the MTT assay. The Fourier transform infrared spectroscopy (FTIR) study showed that there are strong intermolecular hydrogen bonds between the chitosan and polyvinyl alcohol molecules. The Swelling and Porosity tests showed favorable results, obtaining maximum values of $5519 \%$ and $72.17 \%$ respectively. MTT tests determined that the prepared materials are not cytotoxic. These findings suggest that scaffolds possess properties suitable for use in Tissue Engineering.

Keywords: biomaterials; cell control; chitosan; polyvinyl alcohol; scaffold; tissue engineering 


\section{Introduction}

Tissue engineering is an important alternative in the development and future of medicine and focuses on the study and development of biological substitutes to repair or regenerate tissues and organs thanks to biomaterials [1]. A biomaterial is defined as any material used to make devices that replace a body part or function in a safe, reliable, economical, and physiologically acceptable manner [2]. These can be used without incompatibilities in biomedical applications and also to be placed in contact with living tissues without causing damage or alterations. Therefore, they must meet the requirements of the specific application for which they are designed, in addition to presenting high biocompatibility when evaluating their in vitro and in vivo behavior [3]. At present, the use of biomaterials has application in almost all systems of the human organism and there is a wide range of useful materials for all types of biomedical applications. These can be classified according to their chemical nature into metals, polymers, ceramics and composites. Polymers are widely used for their variety of compositions, ease of production with different geometric patterns and with certain properties, they can be rigid or soft and some are suitable for temporary replacements due to their biodegradable composition [4]. Polymeric materials have a wide variety of applications in the biomedical field since they have physical, chemical and mechanical properties that are closer to those of living tissues. In addition, they are easy to process and can be obtained in various forms. Currently, there are numerous polymers used in the biomedical field, among which we can find synthetic and natural ones. The formers are obtained by controlled polymerization processes from low molecular weight raw materials and the latter come directly from nature [5]. One of the strategies used for tissue regeneration is the use of scaffolds, which are highly porous structures that serve as a template to guide the formation of new tissue [6]. The scaffold must have special characteristics that allow it to perform its function without creating an adverse response in the body. These characteristics are biocompatibility with the tissue, biodegradability (at an ideal frequency that corresponds with the formation of the new tissue), zero toxicity, optimal mechanical properties and an adequate morphology that allows the transport of cells, gases, nutrients and metabolites [1]. To develop a scaffold with these properties, a broad type of biodegradable polymers of natural and synthetic origin have been studied. This is because natural polymers are known to have better biological properties than synthetic polymers, and synthetic polymers stand out for their mechanical properties [7]. The combination of these materials is known as Bioartificial Polymeric Systems (BPS) [8]. In this context, for this work a scaffold made of two polymers, chitosan $(\mathrm{CH})$ and polyvinyl alcohol (PVA) was prepared and characterized. $\mathrm{CH}$ is a natural polymer known for its biological properties such as biocompatibility, biodegradability, zero toxicity, and antibacterial properties [7]. An important property of $\mathrm{CH}$ in the context of tissue engineering is the ease with which it can be functionalized. The functional groups present in $\mathrm{CH}$ have the facility to join with other groups, peptides or amino acids, allowing it to combine with other materials, which would improve $\mathrm{CH}$ in different applications of tissue engineering [9]. $\mathrm{CH}$ is considered a biodegradable biomaterial, because it is easily hydrolyzed and metabolized by enzymes (chitosanases and lysozymes) [9]. CH-based scaffolds must meet a series of parameters such as biodegradability, biocompatibility and, 
in the same way, they must mimic the environment of the tissue to be recovered in order to provide an adequate environment where cells can proliferate and differentiate [10]. PVA is a synthetic hydrophilic polymer known for its easy preparation, zero toxicity, biodegradability, high chemical resistance and physical properties. It is produced by partial or total hydrolysis of polyvinyl acetate to remove acetate groups. The amount of hydroxylation determines the physical, chemical, and mechanical properties of PVA. This polymer is highly soluble in water, but resistant to most organic solvents [11]. The higher the degree of hydroxylation and polymerization of PVA, the lower the solubility in water and the more difficult it is to crystallize [12]. Due to its solubility in water, PVA needs to be cross-linked to form scaffolds. These crosslinks, whether chemical or physical, provide the structural stability the scaffold needs after it swells in the presence of water or biological fluids. An advantage in the application of this material is its polarity. due to the fact that it has hydroxyl groups in its chemical structure. These groups tend to form hydrogen bonds between hydroxyl groups, which generates ease in mixing with other materials, obtaining an improvement in the structure [13]. The scaffold was prepared through a process known as lyophilization, this process is one of the most used since the level of porosity can be controlled with the freezing time, after that, the samples are subjected to a vacuum process where the solvent residues, they evaporate by sublimation and leave spaces in the samples known as pores.

Investigations on the use of PVA in the manufacture of scaffolds have been reported due to its hydrophilicity; excellent chemical stability (stable $\mathrm{pH}$ ), which allows cells to grow in a favorable environment; and semipermeability, which favors the transport of oxygen and nutrients necessary for cell survival. In 2005, a study was reported in which scaffolds were made with PVA and alginate (natural hydrophilic and biodegradable polymer) in different concentrations of PVA $(10,30,50 \mathrm{wt} \%)$. The porous bodies were manufactured by a lyophilization process. Porosity values around $85 \%$ were obtained for the three concentrations prepared and pore sizes between 190 and 290um. It was concluded that, from the porosity and pore size values, the scaffold obtained an ideal surface area, in which the cells can interact. The alginate and PVA scaffold were shown to have not only better mechanical properties, but also better adhesion and cell growth than the control scaffold with only alginate [14]. In 2013, another study was carried out where PVA was mixed with two different concentrations $(0.5 \%$ and $4 \%$ ) of gelatin (a biocompatible and biodegradable natural polymer). The scaffold was manufactured through a freeze-drying process. Its degree of swelling was $1500 \%$ for the $4 \%$ concentration, while the $0.5 \%$ concentration obtained an absorption of $1000 \%$ of its original weight. The ideal pore size was obtained with a concentration of $4 \%$, with a range between 100 and $120 \mathrm{um}$. It was concluded that properties such as porosity and pore size increased their values when the gelatin concentration increased, which influences the water absorption capacity of the material [15]. 
On the other hand, $\mathrm{CH}$ has been used to synthesize scaffolds due to its biocompatibility and biodegradability properties. In a study made in 2010, scaffolds with $\mathrm{CH}$ and acetic acid were prepared, varying the $\mathrm{CH}$ concentration by $1.25 \%, 1.5 \%$ and $1.8 \%$. Porous bodies were obtained by lyophilization process. The porosity values obtained were $85 \%, 90 \%$ and $95 \%$ for the concentrations $1.8 \%, 1.5 \%$ and $1.25 \%$, respectively. The effects of variations in the $\mathrm{CH}$ concentration on the morphology of the scaffold were observed through the SEM test, obtaining a pore size range between 50 and 100um. The pore size increased as the $\mathrm{CH}$ concentration decreased. The swelling values were $800 \%, 1200 \%$ and $1600 \%$ for the concentrations of $1.8 \%, 1.5 \%$ and $1.25 \%$, respectively. It was concluded that by having a reduced $\mathrm{CH}$ volume, the scaffold properties improved. Additionally, $\mathrm{CH}$-prepared scaffold has potential in tissue engineering applications [16]. In 2013, $\mathrm{CH}$ was mixed with three different concentrations $(0.0025 \%, 0.005 \%, 0.01 \%)$ of carbon nanotubes (biomaterial with excellent mechanical properties). The scaffold was manufactured through a freeze-drying process. The porosity obtained was $87.7 \%$ for the lowest concentration $(0.0025 \%)$, while increasing the concentration to $0.005 \%$ and $0.01 \%$, values of $88.5 \%$ and $88.8 \%$ were obtained respectively. The degree of swelling was $600 \%$ for the $0.0025 \%$ concentration, while the remaining two concentrations $(0.005 \%$ and $0.01 \%)$ obtained an absorption of $1000 \%$ with respect to their original weight. In the SEM test, the $\mathrm{CH}$ scaffold showed better values when obtaining a pore size between the 100 and 120um range. It was concluded that the $\mathrm{CH}$ concentration allowed the scaffold to have a surface area where the cells can interact [17]. Studies using these two materials have also been reported. In 2015, CH and PVA scaffolds were synthesized with methylcellulose (MC-biodegradable cellulose ether that presents good solubility in water at low temperature). Three concentrations were prepared, varying the amount of MC (25\%,50\% and 75\%) and keeping the amounts of $\mathrm{CH}$ and PVA constant. The method used to manufacture these scaffolds was lyophilization. The degree of swelling increased for the concentration of $25 \%$, the $\mathrm{CH}$ hydroxyl groups and PVA have a positive influence on the improvement of this property. The maximum value of porosity was $88 \%$ for the three concentrations. The morphology of the scaffolds was observed through the SEM test, the pore sizes obtained were 200 and 500um. It was concluded that the scaffold had a porous surface with different pore size distributions, facilitating the entry of nutrients and cell products, which is beneficial for cell growth. Furthermore, with the swelling values obtained, the mechanical properties of the scaffold and the liquid retention capacity can be improved [18].

The combination of these materials has been reported in several studies, and by means of the degree of swelling or swelling tests, porosity, FTIR and cell viability study through the reduction of the MTT compound, the physicochemical and cell viability properties of the prepared scaffold can be described. 


\section{Materials and Methods}

\subsection{Materials}

It was used low molecular weight chitosan (de-acetylation 75\% -85\%), PVA (130,000 g / mol, 99\% hydrolysis), CytoSelect Brand Kit Cell Biolabs, Inc., the cell viability study was done by MTT compound or 3- (4,5-dimethylthiazol-2-yl) -2,5-diphenyltetrazole, and DMEM culture medium (Dubelcco's Modified Eagle Medium) which were purchased from Sigma Aldrich Colombia.

\subsection{Methods}

\subsubsection{Scaffold Synthesis}

A $2 \% \mathrm{~m} / \mathrm{v}$ solution of chitosan (SQT) in 5\% v/ v glacial acetic acid was prepared. $\mathrm{CH}$ was dissolved by continuous stirring for $24 \mathrm{~h}$ on a Heidoplh MR 3001 heating plate. Subsequently, a $2 \% \mathrm{~m} / \mathrm{v}$ solution of PVA (SPVA) was prepared with distilled water at $80^{\circ}$ $\mathrm{C}$ by continuous stirring for $24 \mathrm{~h}$. Both solutions were mixed in three different concentrations, (i) 25\% SQT - 75\% SPVA, (ii) 50\% SQT - 50\% SPVA, (iii) 75\% SQT - 25\% SPVA. Subsequently, the solutions were poured into $2 \mathrm{~cm}$ diameter and $0.5 \mathrm{~cm}$ high cylindrical molds; and they were frozen for $24 \mathrm{~h}$ at $-20^{\circ} \mathrm{C}$. Finally, solutions (i), (ii) and (iii) were subjected to a lyophilization process at approximately 10-2 atm in a Supermodulyo Freeze Dryer brand Thermo Electron Corp lyophilizer in a period of $48 \mathrm{~h}$ until the porous bodies were obtained.

\subsubsection{Characterization tests}

\subsubsection{Fourier Transform Infrared Spectrophotometry (FTIR).}

This test is based on the fact that most molecules absorb light in the infrared range of the electromagnetic spectrum and this energy is converted into molecular vibration. This absorption is specific to the bonds between the atoms present in the molecule. Using a spectrometer, this absorption of infrared light through the sample material is measured as a function of wavelength.

Spectra for $\mathrm{CH}$, pure PVA, and mixtures (i), (ii) and (iii) were obtained using a Thermo Fisher brand Nicolet iS5 spectrometer, equipped with an attenuated total reflectance (ATR) instrument, using a Zn-Se crystal. Each spectrum represents 64 co-aggregated scans referenced against an ATR cell. The range used was 4000 to $650 \mathrm{~cm}-1$ with a resolution of $1.92[19]$. 


\subsubsection{Swelling}

The property of a biomaterial to absorb water influences the conservation of the material's structure and cell growth $[4,20]$. 3 samples were taken from each specimen, for a total of 9 samples evaluated. These were weighed after the freeze-drying process using a KERHN \& Sohn $\mathrm{GmbH}$ analytical balance model D-72336. Subsequently, each specimen was immersed in distilled water at $20^{\circ} \mathrm{C}$ and every $3 \mathrm{~s}$ they were removed, the surface moisture was dried with filter paper and $W f$ was weighed immediately. Finally, to determine the degree of swelling, the following equation was used:

$$
W_{H}(\%)=\left(\frac{W_{f}-W_{i}}{W_{f}}\right) * 100
$$

Where:

WH Percent swelling of the sample.

Wf Sample weight after being dipped.

$W i \quad$ Initial sample weight.

\subsubsection{Porosity}

Porosity plays an important role in scaffold synthesis because pores provide nutrition for cells and tissue [21]. The percentage of porosity was calculated as follows:

$$
P(\%)=\left(\frac{V_{1}-V_{3}}{V_{2}-V_{3}}\right) * 100
$$

Where:

V1 Volume of distilled water used.

$V 2$ Total volume of distilled water after submerging the sample.

$V 3$ Volume of distilled water remaining after sample removal.

$P \quad$ Porosity percentage.

2.2.2.4 Assay of cell viability (metabolic activity) by reduction of compound MTT.

The cell viability test is divided into two parts:

Sterilization of scaffolds. In order to avoid the presence of bacteria or microorganisms acquired from the medium that could alter the result of the assay, specimens (i), (ii) and (iii) were placed in 12-well NEST Biotech brand sterile plates, where there were added 3 series of $2 \mathrm{~mL} \mathrm{v} / \mathrm{v}$ of ethanol at concentrations of $70 \%, 50 \%$ and $30 \%$. Each ethanol series was 
added at $12 \mathrm{~h}$ intervals. Subsequently, PBS was added to each specimen in order to obtain a neutral $\mathrm{pH}$, and a medium in which the cells can survive.

Cell culture and MTT. For cell culture, cells were trypsinized, centrifuged, and resuspended in DMEM culture medium. 12-well plates were used, in which osteoblasts were incubated. The incubation process was carried out in a BINDER incubator at $37^{\circ} \mathrm{C}$ and $5 \% \mathrm{CO} 2$. After $48 \mathrm{~h}$ of incubation, MTT solution was added to each well, and incubated again for 100 to 120 min. Subsequently, all the suspended medium in each well was removed and DMSO detergent was added, in order to solubilize the formazan crystals that form once the MTT is added. The absorbance values were measured in a Biotek Model 800 TS ELISA reader at 590 $\mathrm{nm}$ [22].

\section{Results and discussion}

\subsection{Characterization tests}

\subsubsection{FTIR Characterization}

The FTIR characterization was used to evaluate the functional groups of the polymers. Figure 3 shows the FTIR spectrum of low molecular weight QT. In the band from 3000 to $2840 \mathrm{~cm}-1$ the narrowing of the $\mathrm{C}-\mathrm{H}$ bonds of the alkyl groups is evidenced. The peaks observed in the range from 1651 to $1346 \mathrm{~cm}-1$ show the presence of amide I, amide II and amide III, which are characteristic groups of QT. Also, the pronounced peaks at 1068 to $889 \mathrm{~cm}-1$ demonstrate the narrowing of C-O bonds present in the QT [20]. 


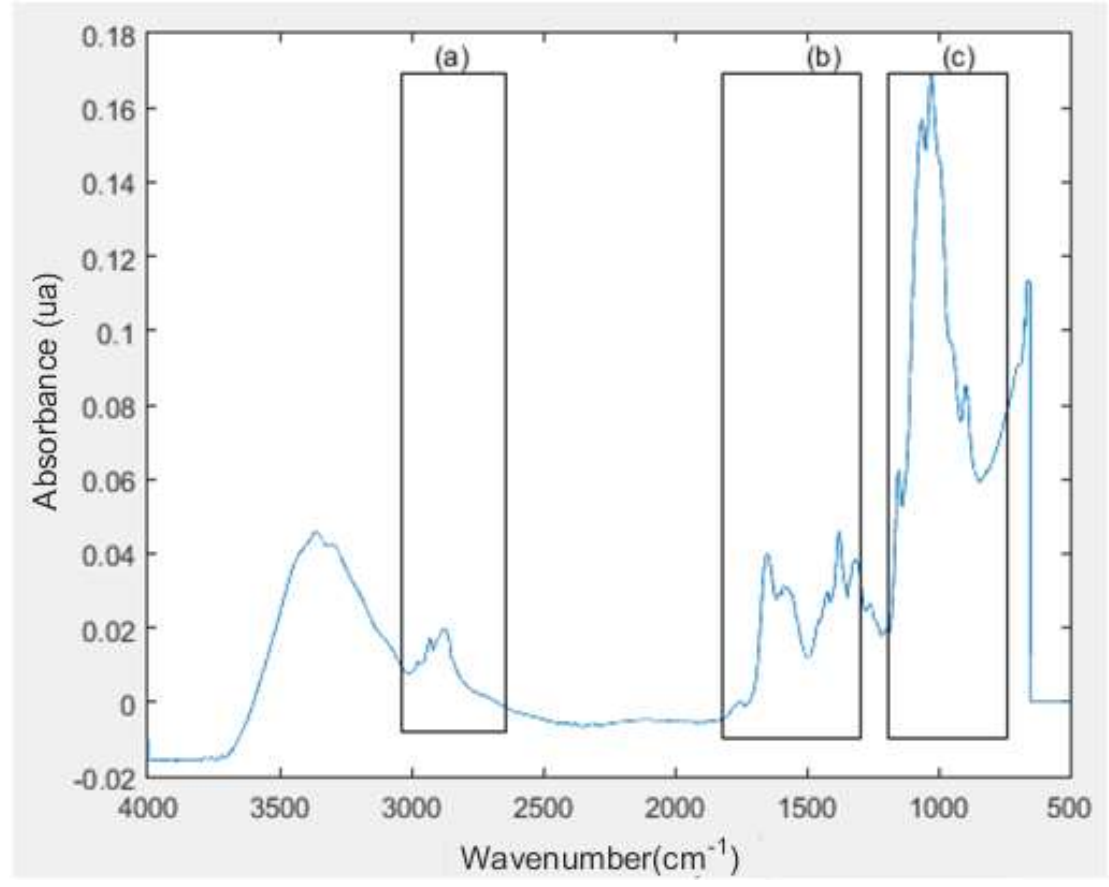

Figure 3. Low molecular weight chitosan spectrum

Figure 4 shows the FTIR spectrum for pure PVA. In the range of 3447 to $3200 \mathrm{~cm}-1$, the narrowing of the $\mathrm{O}-\mathrm{H}$ groups can be observed, due to the intermolecular and intramolecular forces of hydrogen bonds [20]. The band observed between 3000 and $2840 \mathrm{~cm}-1$ is the result of the narrowing of $\mathrm{CH}$ bonds, and the peaks shown between 1730 and $1680 \mathrm{~cm}-1$ are due to the narrowing of $\mathrm{C}=\mathrm{O}$ and $\mathrm{CO}$ bonds of the remaining acetate groups of the PVA (reaction of saponification of polyvinyl acetate) [20]. 


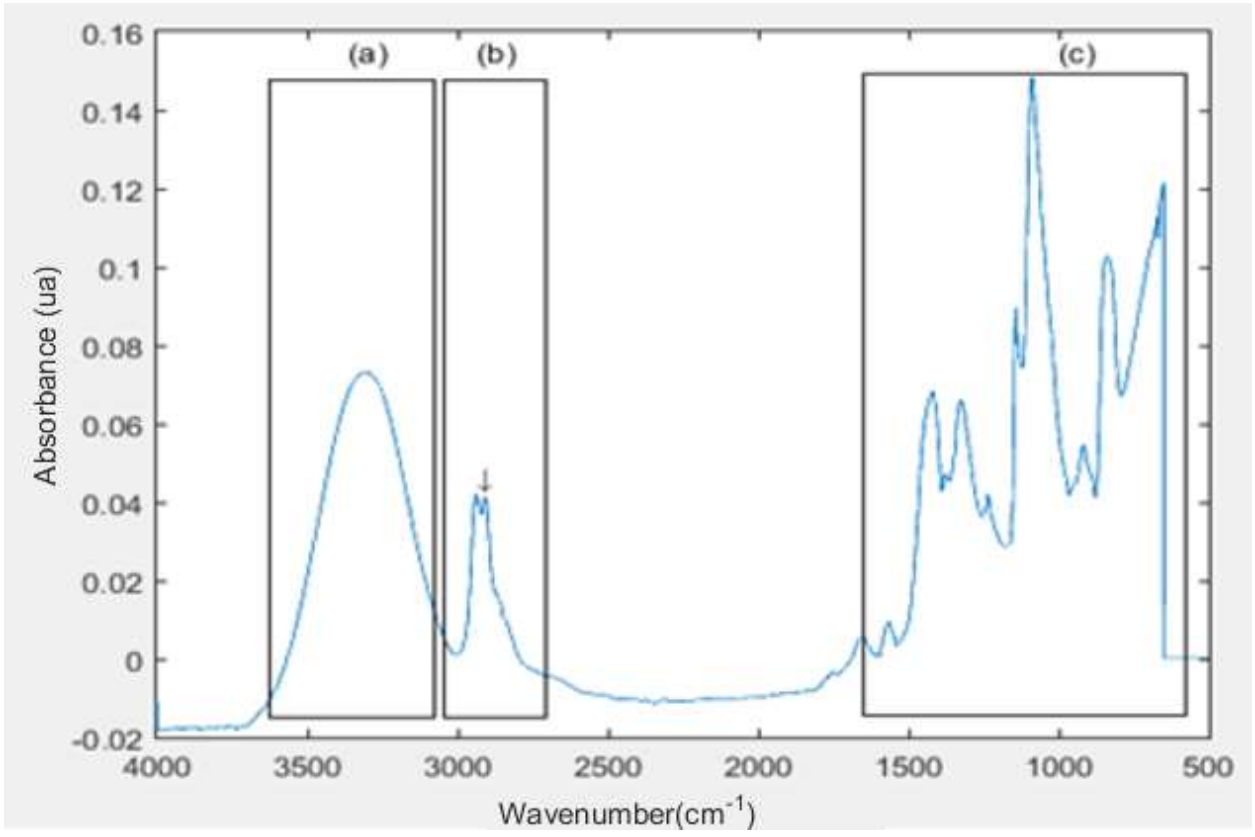

Figure 4. Infrared spectrum of polyvinyl alcohol

Figure 5 shows the FTIR spectrum corresponding to specimens (i), (ii) and (iii). In the range of 3100 to $2800 \mathrm{~cm}-1$, stretching of $\mathrm{C}-\mathrm{H}$ bonds is observed. In the range of 3447 to $3200 \mathrm{~cm}-$ 1 , the vibrations of the $\mathrm{N}-\mathrm{H}$ and $\mathrm{C}-\mathrm{H}$ groups can be observed $[5,20]$. Each interval can be seen in sections (a) and (b) of Figure 5.

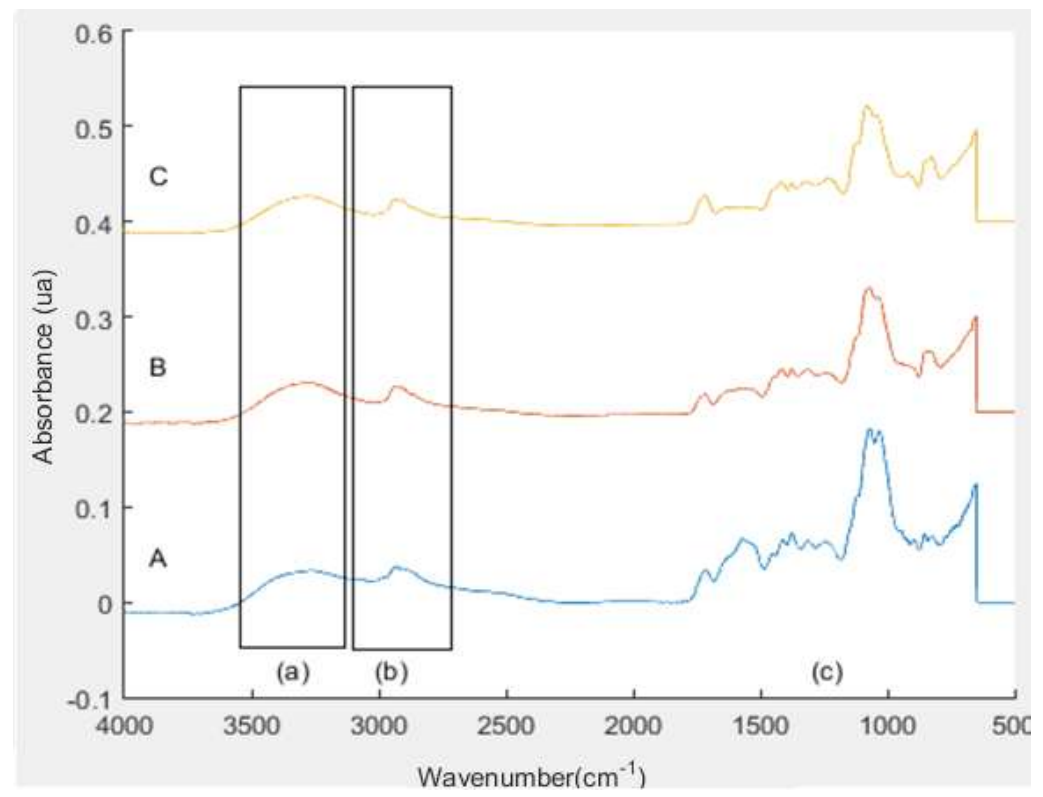

Figure 5. FTIR PVA + Chitosan. A. 75\% QT - 25\% PVA; B. 50\% QT - 50\% PVA; C. 25\% QT 75\% PVA. (a) N-H and O-H groups; (b) Stretching C-H bonds. 


\subsubsection{Swelling Characterization}

One of the most relevant aspects to consider in the study of scaffolds is their maximum degree of water absorption, since the capacity to absorb and retain water is directly related to the capacity to diffuse nutrients and oxygen [23].

In Figure 6 the swelling percentage of the scaffolds can be observed for the specimens (i) $75 \% \mathrm{CH}-25 \%$ PVA, (ii) $50 \% \mathrm{CH}-50 \%$ PVA and (iii) $25 \% \mathrm{CH}-75 \%$ PVA. The three specimens absorbed a large percentage of water with respect to their weight in the initial 30s, with values of $4839 \%, 3980 \%$ and $3589 \%$ for (i), (ii) and (iii), respectively. Likewise, after 60 s, the swelling percentage for each specimen reached a maximum of $5519 \%, 4148 \%$ and $3775 \%$ for (i), (ii) and (iii), respectively. The results show that $\mathrm{CH}$ greatly influences the swelling volume and reduces it from $5519 \%$ to $3775 \%$ when mixed with PVA. The reduction in the swelling volume can be attributed to a more rigid network formed by the intermolecular and intramolecular reactions of the polymers [24]. The increase in the swelling value can be explained through the high availability of amino groups, responsible for non-covalent bonds in water, which occupy the free space between chains and increase the volume of the sample. The degree of reduction also depends on factors such as the molecular weight of the components, $\mathrm{pH}$, temperature [25].

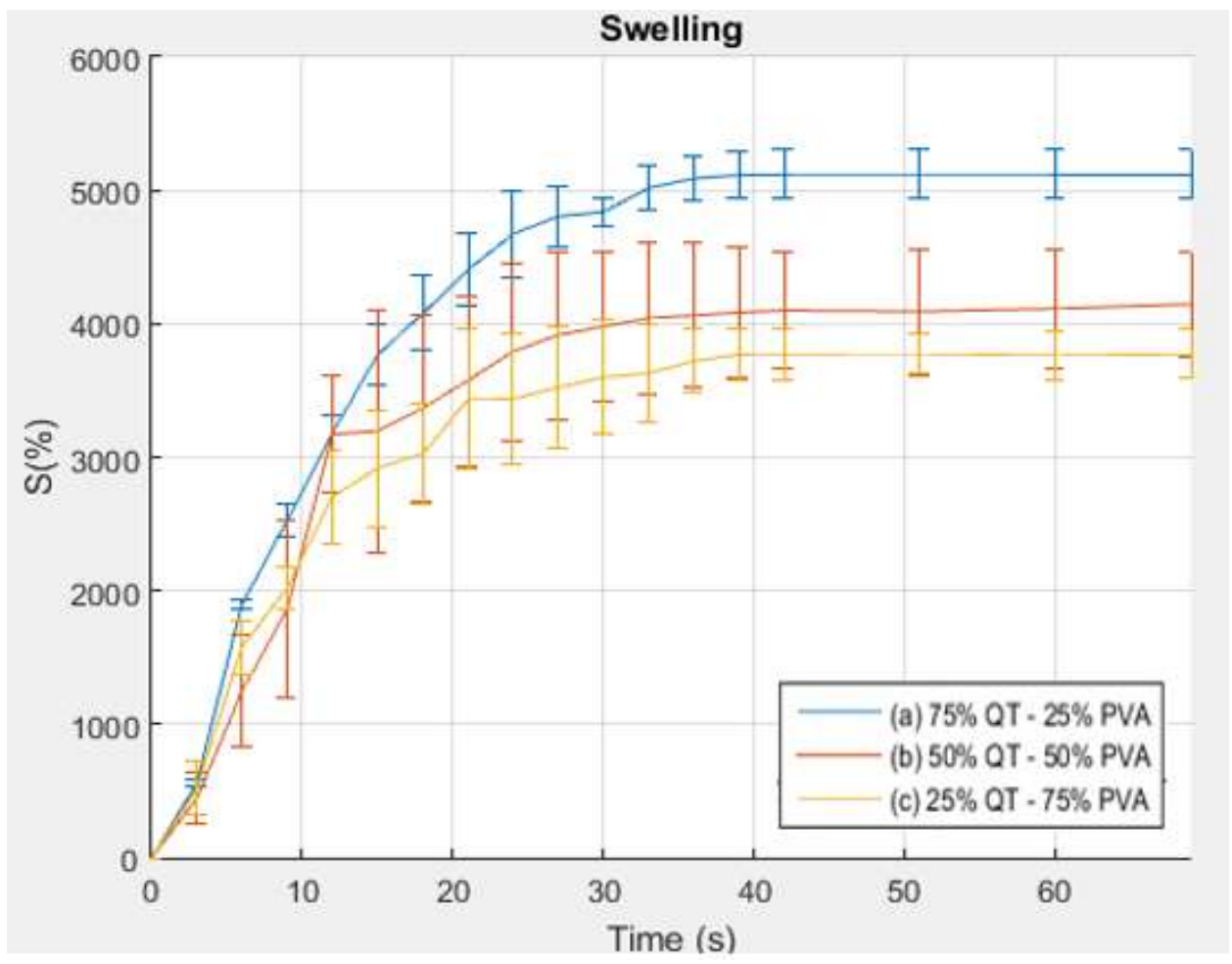

Figure 6. Swelling curve of the prepared scaffolds. 
Table 1. Percentage of swelling specimens evaluated

\begin{tabular}{ccc}
\hline Samples & Time (s) & Swelling (\%) \\
\hline $75 \% \mathrm{CH}-25 \%$ PVA & 60 & $5519 \pm 956$ \\
$50 \% \mathrm{CH}-50 \%$ PVA & 60 & $4148 \pm 720$ \\
$25 \% \mathrm{CH}-75 \%$ PVA & 60 & $3775 \pm 538$ \\
\hline
\end{tabular}

\subsubsection{Porosity Characterization}

Porosity plays an important role in scaffolds used in tissue engineering because pores allow cell migration and blood vessel growth through the scaffold. Furthermore, pores ensure the effective exchange of nutrients and waste between cells and their microenvironment [59]. The porosity found for specimens (i), (ii) and (iii) is shown in Table 1. It can be seen that the maximum porosity was of the specimen (i), with the highest QT concentration. Likewise, the minimum value of porosity was noted in the specimen (ii), with an equivalent concentration of QT and PVA. No greater difference was obtained in the porosity percentages of the specimens (i), (ii) and (iii), this is due to the freeze-drying process used in the synthesis of the scaffold. In the literature it can be found that when handling more cycles in lyophilization, the percentage of porosity increases [14], while in the methodology of the present investigation, a lyophilization cycle was used for all specimens. This is related since the samples at the time of being frozen are subjected to an increase in pressure and the solvent passes from the liquid to the gas phase, leaving a space where the pore is formed [14]. Generally, a high degree of porosity is a characteristic of an ideal scaffold used in tissue engineering. A large space and nutrition for the cells will be given by a scaffold with a high level of porosity [24].

Table 2. Percentage of porosity evaluated specimens

\begin{tabular}{cc}
\hline Samples & Porosity (\%) \\
\hline $75 \% \mathrm{CH}-25 \%$ PVA & $72.17 \pm 4.56$ \\
$50 \% \mathrm{CH}-50 \%$ PVA & $70.12 \pm 2.30$ \\
$25 \% \mathrm{CH}-75 \%$ PVA & $70.19 \pm 0.38$ \\
\hline
\end{tabular}




\subsubsection{Cell viability assay (metabolic activity) by reduction of compound MTT}

In tissue engineering, an ideal scaffold is the one that in addition to having excellent mechanical properties, does not produce toxic or adverse effects when applied to living tissue. The effect of scaffolds on osteoblast proliferation and survival viability was studied by means of the MTT compound reduction cell viability assay.

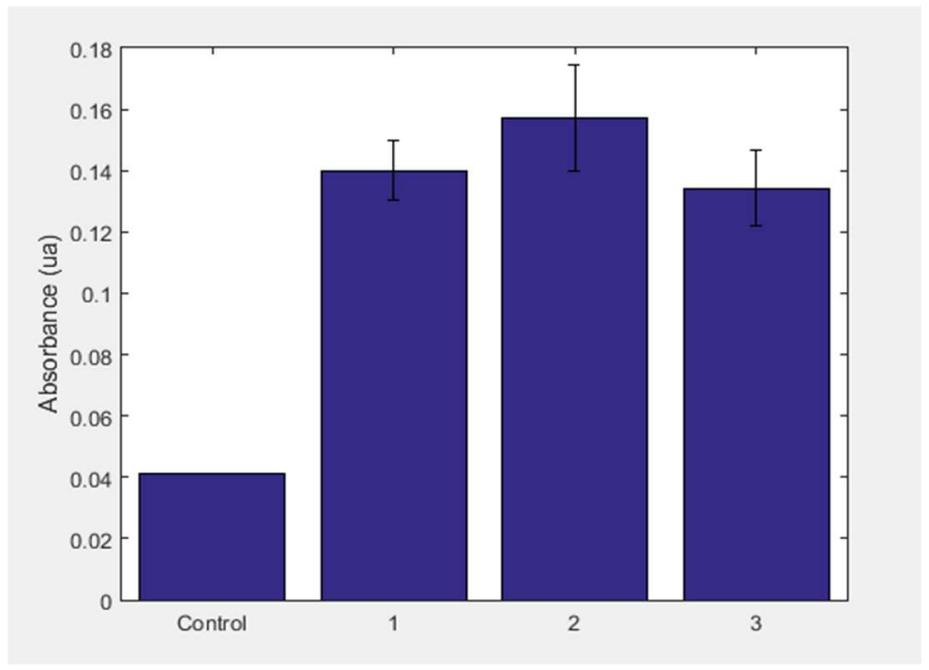

Figure 7. MTT test results.

The absorbance level and standard deviation obtained for each specimen can be seen in figure 7. Each absorbance value is the average of the absorbance values in each well. These values were obtained from the ELISA reader. It can be seen that the absorbance value for specimens (i), (ii) and (iii) is considerably higher than that of the control, which demonstrates the presence of cells adhered to the scaffold at the end of the assay (Figure 8). The absorbance values of the different specimens did not show greater variation. Furthermore, it can be seen that the lowest absorbance value was obtained in the specimen with the highest quantity of QT. This can be attributed to the shape of the pores that the scaffold may have, which influences adhesion, proliferation and migration of cells [20]. Likewise, the high absorbance of the specimens can be explained due to the high level of biocompatibility that QT has with osteoblasts [7], and an adequate sterilization process. 


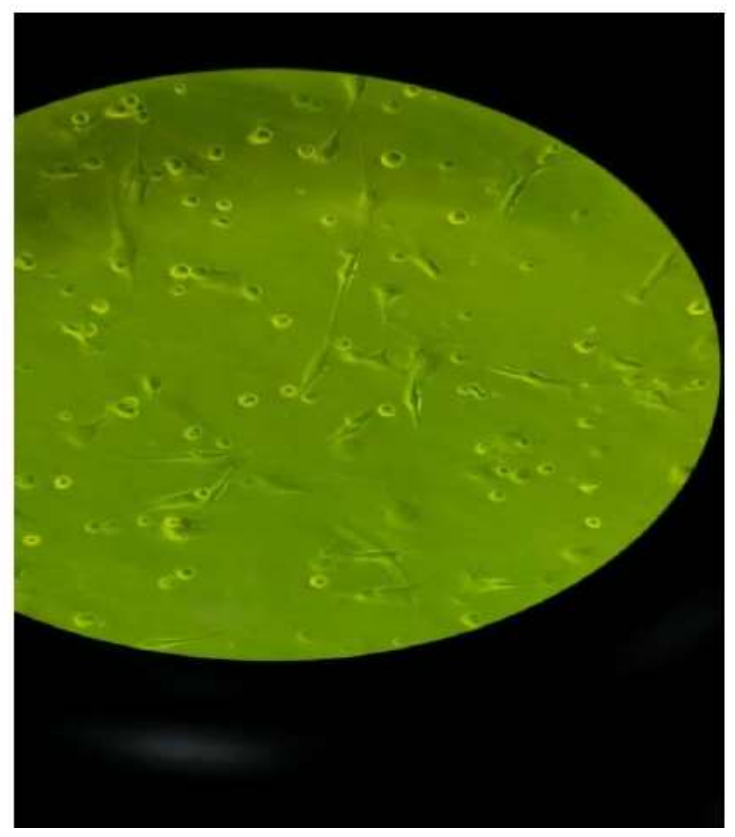

Figure 8. Cell culture concentration (i)

\section{Conclusions}

In this investigation, a scaffold was synthesized with two polymers $\mathrm{CH}$ and PVA. The study showed that by varying the concentration of QT and PVA, associated with the lyophilization method used, it is possible to control properties that will allow the scaffold to be applied in bone regeneration.

In the first place, the values obtained in porosity were similar because a lyophilization stage was used for the three samples, a process which allows controlling the values of this property. Additionally, the results of the degree of swelling indicate that in a concentration with a higher quantity of $\mathrm{CH}$, the scaffold obtains better swelling properties. This result is consistent with others in the literature, and it is demonstrable because there is less presence of hydrophilic groups in a PVA-QT mixture, when the concentration of PVA is increased.

As mentioned above, the MTT assay was performed in order to quantify the formation of formazan crystals through the changes in absorbance that the samples presented at a wavelength of 590nm, values that proportionally represent cell viability. First, the scaffold had to be sterilized, so that the cell viability assay was not affected. This was achieved by means of series of ethanol and PBS, and it proved to be an effective method to achieve a sterile environment in which the cells can survive, due to the fact that no turbulence or color changes were observed in the medium where the cells were grown. Furthermore, it is a simple procedure which can be implemented in future research. The three specimens showed the presence of cells at the end of the test, the variation in absorbance did not reflect significant changes, which allows us to say that the cells managed to proliferate in a favorable environment. 
Finally, the three specimens were characterized through these tests, however, the one with the highest amount of $\mathrm{CH}$ presented better properties than the others, which means that this research can be expanded by adding more characterization tests that allow to know more thoroughly the properties of an SPBA when synthesized with different concentrations of QT and PVA. It is recommended to study the degradation index of the scaffolds, and a SEM test in order to know more about the structure of the pores.

Author Contributions: H.R.S.P., A.V.B., D.N.M., D.M.F. Collaboration with the preparation of cell viability tests and laboratory material; A.B.B. Supervision of methodology and writing-original draft preparation; S.R.T., J.O.M., J.A.G. Were responsible of carrying out the synthesis and characterization phases of the samples and final writing of the manuscript. All authors have read and agreed to the published version of the manuscript.

Funding: This research received no external funding.

Acknowledgments: The authors thank the Manuela Beltran University and Distrital University of Bogota for providing equipment and software to successfully conclude this research.

Conflicts of Interest: The authors declare no conflict of interest.

\section{References}

1. Archana, D.; Upadhyay, L. Chitosan-pectin-alginate as a novel scaffold for tissue engineering applications. Indian journal of Biotechnology. 2013, 12, 475-482.

2. Guvendiren, M.; Molde, J.; Soares, R.; Kohn, J. Designing Biomaterials for 3D Printing. ACS Biomaterials Science \& Engineering, 2016, 2, 1679-1693. DOI: 10.1021/acsbiomaterials.6b00121.

3. Grum. Book Review: Mechanical Properties of Ceramics and Composites Grain and Particle Effects. International Journal of Materials and Product Technology, 2016, 27, 291.

4. Chawla, K. Composite materials 1980: a report on the 3rd International Conference on composite materials, Paris, August 26-29, 1980, Materials Science and Engineering, vol. 48, no. 1, pp. 137-141, 1981.

5. Navarro, M. Desarrollo y Caracterización de Materiales Biodegradables para Regeneración Ósea. Universitat Politècnica de Catalunya. Departamento de Ciencia de los Materiales e Ingeniería Metalúrgica. Barcelona - España, 2005.

6. Thein-Han, W.; Misra, R. Biomimetic chitosan-nanohydroxyapatite composite scaffolds for bone tissue engineering. Acta Biomaterialia,2009,5,1182-1197. 
7. Kanimozhi, K.; Khaleel, S.; Sugantha, V. Processing and characterization of chitosan/PVA and methylcellulose porous scaffolds for tissue engineering. Materials Science and Engineering.2016, 61, 484-491.

8. Bernal-Ballen, A.; Lopez-Garcia, J.; Merchan-Merchan, M.; Lehocky, M. Synthesis and Characterization of a Bioartificial Polymeric System with Potential Antibacterial Activity: Chitosan-Polyvinyl Alcohol-Ampicillin. Molecules, 2018,23,3109.

9. Amidi, M; Mastrobattista, E; Jiskoot, W.; Hennink, W. Chitosan-based delivery systems for protein therapeutics and antigens. Advanced Drug Delivery Reviews, 2010,62,5982.

10. Wan, Y.; Wu, H.; Cao, X.; Dalai, S. Compressive mechanical properties and biodegradability of porous poly(caprolactone)/chitosan scaffolds. Polymer Degradation and Stability, 2008, 93, 1736-1741.

11. Tubbs, R. Sequence distribution of partially hydrolyzed poly(vinyl acetate). Journal of Polymer Science Part A-1: Polymer Chemistry, 1966, 4, 623-629.

12. Jones, J. Polyvinyl alcohol. Properties and applications, British Polymer Journal, 1973, 5, 493-494.

13. Kumar, A.; Han, S. PVA-based hydrogels for tissue engineering: A review. International Journal of Polymeric Materials and Polymeric Biomaterials, 2016,66,159-182.

14. Cho, S.; Lee, J. Fabrication and characterization of porous alginate/polyvinyl alcohol hybrid scaffolds for 3D cell culture. Journal of Biomaterials Science, 2005,16,933-947.

15. Choi, D.; Singh, A.; Kumar, T.; Cho and S. Han. Porous Three-Dimensional PVA/Gelatin Sponge for Skin Tissue Engineering. International Journal of Polymeric Materials, 2013,62,384-389.

16. Yang, B. Preparation and characterization of a novel chitosan scaffold. Carbohydrate Polymers, 2010, 80, 860-865.

17. Venkatesan, B.; Ryu, P.; Sudha and S. Kim.Preparation and characterization of chitosan-carbon nanotube scaffolds for bone tissue engineering. International Journal of Biological Macromolecules, 2012, 50, 393-402.

18. Kanimozhi, V.; Khaleel, S.; Kumari, V. Processing and characterization of chitosan/PVA and methylcellulose porous scaffolds for tissue engineering. Materials Science and Engineering,2016,61,484-491.

19. Rajeswari, A.; Amalraj, A.; Pius, A. Removal of phosphate using chitosan-polymer composites. Journal of Environmental Chemical Engineering.2015, 3, 2331-2341.

20. Mozafari, M. Synthesis and characterization of electrospun polyvinyl alcohol nanofibrous scaffolds modified by blending with chitosan for neural tissue engineering. International Journal of Nanomedicine, 2012,7,25.

21. Pandey, E.; Srivastava, K.; Gupta, S.; Mishra, N. Some biocompatible materials used in medical practices- a review. International Journal of Pharmaceutical Sciences and Research, 2016,2748-2755.

22. Yang, X.; Yang, K.; Wu, S.; Chen, X.; Yu, F.; Li, J.; Zhu, Z. Cytotoxicity and wound healing properties of PVA/ws-chitosan/glycerol,2010.

23. Compan V.; Guzmán J.; Riande E. A potentiostatic study of oxygen transmissibility and permeability through hydrogel membranes. Biomaterials. 1998;19(23):2139-45 
24. Kanimozhi, K.; Basha, S.; Kumari. Development of Biomimetic Hybrid Porous Scaffold of Chitosan/Polyvinyl Alcohol/Carboxymethyl Cellulose by Freeze-Dried and Salt Leached Technique. Journal of Nanoscience and Nanotechnology, 2018,18(7), 4916-4922.

25. El-Hefian, E.; Nasef, M. The Preparation and Characterization of Chitosan / Poly (Vinyl Alcohol) Blended Films. E-Journal of Chemistry, 2010,7(4), 1212-1219. 